\title{
Leptogenesis and low energy neutrino data
}

\author{
Pasquale Di Bari*广 \\ Physics and Astronomy, University of Southampton \\ E-mail: P.Di-Bari@soton.ac.uk
}

I will discuss some recent results on leptogenesis showing how even high scale minimal leptogenesis scenarios, thermal and based on type I see-saw mechanism, can be tested with low energy neutrino data. We will mainly focus on $S O(10)$-inpsired leptogenesis scenarios leading to interesting predictions that already partly start to be (positively so far) tested by the experimental results.

Frontiers of Fundamental Physics 14 - FFP14,

15-18 July 2014

Aix Marseille University (AMU) Saint-Charles Campus, Marseille

\footnotetext{
* Speaker.

$\dagger$ I am indebted to all my collaborators who contributed to the results presented in this talk: Alexey Anisimov, Stefen Antusch, Enrico Bertuzzo, Wilfried Buchmüller, Steve Blanchet, Ferruccio Feruglio, David Jones, Steve F. King, Sophie King, Luca Marzola, Michael Plümarcher, Georg Raffelt, Michele Re Fiorentin, Antonio Riotto.
} 


\section{Introduction}

Leptogenesis [1,2] can be regarded as a bridge between the physics of the early Universe and neutrino physics. On one side it aims at establishing a new important stage in the history of the Universe solving the problem of the origin of the matter-antimatter asymmetry. On the other side it can be regarded as a complementary phonemenological tool, sensitive to those high energy see-saw [3] neutrino parameters that escape conventional tests from low energy neutrino experiments.

One would ideally like to answer two important questions: The first one is whether with leptogenesis we can understand the existing information on neutrino masses and mixing and hopefully predict the unknown parameters; Vice-versa one would like also to understand whether low energy neutrino experiments could be sufficient to test leptogenesis.

In the LHC era typical answers to these questions are negative (in both cases) and one needs actually to lower the scale of leptogenesis in order to have a testable model with signatures at the LHC or in (charged) lepton flavour violating processes or with enhanced electric dipole moments of electron or neutron or other kinds of phenomenological new connected prediction.

Should one then completely give up the possibility of a traditional high energy scale model of leptogenesis? This question is particularly appropriate now considering on one hand the negative results from the first stage of the LHC and from all other efforts to find new physics at the $\mathrm{TeV}$ (or lower energy) scale (including Dark Matter searches that might be directly or indirectly related to low scale models of leptogenesis) and on the other hand the discovery of a non-vanishing reactor neutrino mixing angle that has opened the door to a completion of the measurements of the leptonic mixing matrix parameters increasing the opportunities for models that do not necessarily predict new observables beyond the low energy neutrino parameters. From this point of view one should also include the great efforts and improved upper bounds on the absolute neutrino mass scales that make quite plausible the detection of a signal within next ten years especially from cosmology.

Current low energy neutrino experiments measure three mixing angles within the following $3 \sigma$ ranges [4]

$$
\begin{aligned}
& \theta_{13}=7.6^{\circ}-9.9^{\circ} \text { and } 7.7^{\circ}-9.9^{\circ}, \\
& \theta_{12}=30.6^{\circ}-36.8^{\circ} \text { and } 30.6^{\circ}-36.8^{\circ}, \\
& \theta_{23}=37.7^{\circ}-52.3^{\circ} \text { and } 38.1^{\circ}-52.3^{\circ}
\end{aligned}
$$

respectively for so called normally ordered (NO) and inversely ordered (IO) neutrino mass schemes that we are going to explain in a moment. At $3 \sigma$ any value of the Dirac phase $\delta$ is still allowed, though global analyses tend to favour within $2 \sigma$ values with $\sin \delta<0$. Neutrino oscillation experiments are not sensitive to the nature of neutrinos and so there is currently no information whether neutrino are Majorana or Dirac and therefore there is no information not only on value of the Majorana phases but even if these can be physically observable at all.

At the same time neutrino oscillations experiments also fix two mass squared differences that, for historical reasons, are called the solar mass squared difference $\Delta m_{\mathrm{sol}}^{2}$ and the atmospheric mass squared difference $\Delta m_{\mathrm{atm}}^{2} \gg \Delta m_{\mathrm{sol}}^{2}$. If we label the three neutrino masses in a way that $m_{1} \leq m_{2} \leq$ $m_{3}$, then there are two possibilities: either $\Delta m_{\text {sol }}^{2} \equiv m_{2}^{2}-m_{1}^{2}$ and $\Delta m_{\mathrm{atm}}^{2} \equiv m_{3}^{2}-m_{2}^{2}$ or $\Delta m_{\mathrm{sol}}^{2} \equiv$ $m_{3}^{2}-m_{2}^{2}$ and $\Delta m_{\mathrm{atm}}^{2} \equiv m_{2}^{2}-m_{1}^{2}$. The solar neutrino mass scale is then defined as $m_{\mathrm{sol}} \equiv \sqrt{\Delta m_{\mathrm{sol}}^{2}} \simeq$ 
$0.0087 \mathrm{eV}$ while the atmospheric neutrino mass scale $m_{\mathrm{atm}} \equiv \sqrt{\Delta m_{\mathrm{sol}}^{2}+\Delta m_{\mathrm{atm}}^{2}} \simeq 0.05 \mathrm{eV}$. Since $m_{\mathrm{atm}} \simeq 5 m_{\mathrm{sol}}$, in the first case one has that for $m_{1} \ll m_{\text {sol }}$ the neutrino masses are hierarchical with $m_{3} \gg m_{2} \gg m_{1}$, while in the second case, in the limit $m \ll m_{\text {atm }}$ one has $m_{3} \simeq m_{2} \gg m_{1}$ so called inverted hierarchy. Neutrino oscillations do not give any information on the absolute neutrino mass scale and in particular on the lightest neutrino mass $m_{1}$ and, therefore, more generally the first case should be called normal ordering (NO) and the second case inverted ordering (IO). If $m_{1} \ll m_{\text {sol }}$ neutrinos are hierarchical (normally or inverted) while if $m_{1} \gg m_{\text {atm }}$ the neutrino masses are necessarily quasi-degenerate with $m_{1} \simeq m_{2} \simeq m_{3}$.

The most stringent upper bound on the absolute neutrino mass scale is placed today by cosmological observation. The Planck collaboration has recently set an upper bound on the sum of the neutrino masses $\sum_{i} m_{i} \lesssim 0.23 \mathrm{eV}(95 \%$ C.L.) [5], that combined with neutrino oscillation experiments translates into $m_{1} \lesssim 70 \mathrm{meV}$, both for NO and for IO.

\section{The minimal scenario of leptogenesis}

The minimal scenario of leptogenesis is based on the type I see-saw mechanism and on a thermal production of the heavy right-handed $(\mathrm{RH})$ neutrinos whose (out-of-equilibrium) decays are responsible for the generation of the asymmetry. The SM Lagrangian is augmented by the introduction of RH neutrinos with Yukawa coupling and a Majorana mass term M. After spontaneous symmetry breaking a Dirac mass term $m_{D}$ is generated by the Higgs vev. In the see-saw limit, $M \gg m_{D}$, the spectrum of mass eigenstates splits into a light set of three (almost exactly) left-handed ( $\mathrm{LH})$ neutrinos with masses given by the see-saw formula,

$$
\operatorname{diag}\left(m_{1}, m_{2}, m_{3}\right)=U^{\dagger} m_{D} M^{-1} m_{D}^{T} U^{\star},
$$

and into a heavy set of (almost exactly) RH neutrinos with masses $M_{1} \leq \ldots \leq M_{N}$ (almost exactly) coinciding with the Majorana mass matrix eigenvalues. The minimum number of RH neutrinos $N$ is 2 in order for the see-saw formula to be able to reproduce correctly the experimental data since these indicate so far, as we have seen, the existence of at least two mass scales (the solar and the atmospheric). There is in principle no maximum number but for definiteness we will refer to the case $N=3$ that, as we will see, is nicely justified by $S O(10)$-inspired models.

Importantly, both the heavy and the light neutrino eigenstates are predicted to be Majorana neutrinos. Therefore, one expects that $0 v \beta \beta$ neutrino decay can indeed occur even though, for the NO case, its rate might not be necessarily large enough to be detected by any future experiment. If $T_{R} \gtrsim(2-5) M_{i}$, then the RH neutrino are produced thermally in the early Universe if the Yukawa couplings are not too small. In this case the RH neutrinos decay in general with a different rate into leptons and anti-leptons in way to generate a $B-L$ asymmetry in the form of a lepton asymmetry. For $T_{R} \gtrsim 100 \mathrm{GeV}$ the $B-L$ asymmetry sphalerons processes are in equilibrium in a way that the part of the $B-L$ asymmetry, approximately one third, is converted into a baryon asymmetry. Therefore, leptogenesis predicts an initial temperature in the early Universe many order of magnitudes above that one required by Big Bang Nucleosynthesis $(\sim \mathrm{MeV})$. If one translates the final $B-L$ asymmetry $N_{B-L}^{\mathrm{f}}$ produced by leptogenesis into the usual baron-to-photon number ratio $\eta_{B}$ this is given by $\eta_{B} \simeq a_{\text {spy }} N_{B-L}^{\mathrm{f}} / N_{\gamma}^{\text {rec }}$, where $a_{\text {sph }} \simeq 1 / 3$ is the fraction of $B-L$ asymmetry ending 
up into a baryon asymmetry due to the sphaleron conversion, while $N_{\gamma}^{\text {rec }}$ is the number of photons at the recombination time into a certain portion of co-moving volume properly chosen. The ratio does not depend on this particular choice. The value of $\eta_{B}$ calculated in leptogenesis has then to be compared with the observed valued that from $\mathrm{CMB}$ temperature anisotropies is very accurately and precisely measured, $\eta_{B}^{C M B}=(6.1 \pm 0.1) \times 10^{-10}[5]$.

In general, the see-saw mechanism contains too many parameters to be tested in a model independent way (eighteen in the case $N=3$ ). The reason is that the high energy parameters related to the properties of the heavy neutrinos are inserted by hand into the see-saw Lagrangian if this is not embedded within a model. From this point of view the leptogenesis bound can provide a phenomenological tool sensitive to the properties of the heavy RH neutrinos and hopefully able to over-constraining the see-saw parameter space producing predictions on the lo energy neutrino parameters. Clearly however just one constraint does not seem sufficient to fix the nine parameters associated to the properties of the three RH neutrinos. One has then to introduce some additional conditions able to reduce the number of parameters. The set of conditions specifies the specific leptogenesis scenario that can hopefully produced some predictions on the low energy neutrino parameters.

So called vanilla leptogenesis [2] provides a traditional example of a scenario where the reduction of the number of parameters allows to get an experimental testable prediction. This is the set of the assumptions: (i) the final asymmetry unaffected by the flavour compositions of leptons produced in the decays of the RH neutrinos; (ii) hierarchical RH neutrino spectrum $M_{2,3} \gtrsim 3 M_{1}$; (iii) strong lightest RH neutrino wash-out; (iv) absence of fine tuned cancellations in the see-saw formula; (v) validity of simple Boltzmann equations for the calculation of the symmetry. Under these conditions the final asymmetry is dominated by the contribution of the lightest RH neutrino $N_{1}$ in way that the baryon-to-photon number ratio can be simply calculated as $\eta_{B} \simeq 0.01 \varepsilon_{1} \kappa^{\mathrm{f}}\left(K_{1}\right)$ [6], where $\varepsilon_{1}$ is the lightest RH neutrino total $C P$ asymmetry and $\kappa^{\mathrm{f}}\left(K_{1}\right)$ is the (final value of the) efficiency factor that gives the number of RH neutrinos decaying out-of-equilibrium. It takes into account not only how efficiently RH neutrinos are produced but also what fraction decays out-ofequilibrium, i.e. whose decays are not balanced by inverse processes.

It depends only on the total decay parameter $K_{1}$, the ratio between the lightest RH neutrino decay width and the expansion rate of the Universe at the time when the RH neutrinos become non-relativistic. i.e. when $T \simeq M_{1}$. It is a crucial parameter since it sets the strength of the washout: for $K_{1} \gtrsim 1$ the wash-out is strong and cannot be neglected, for $K_{1} \lesssim 1$ it is weak and can be neglected. Interestingly $K_{1}$ can be written as a linear combination of the light neutrino masses,

$$
K_{1}=\sum_{k} \frac{m_{k}}{m_{\star}}\left|\Omega_{i k}\right|^{2}, \quad \text { where } m_{\star} \equiv \frac{16 \pi^{5 / 2} \sqrt{g_{\star}}}{3 \sqrt{5}} \frac{v^{2}}{M_{\mathrm{Pl}}} \simeq 1.08 \mathrm{eV},
$$

is the so called equilibrium neutrino mass and $\left|\Omega_{i k}\right|=\mathscr{O}(1)$ in the absence of fine tuned cancellations in the see-saw formula. It is quite impressive that given the solar and atmospheric neutrino mass scales the value of $K_{1}$ typically lies in the range $10-50$ corresponding to a wash-out that is strong but not strong enough to repent successful leptogenesis.

Actually such a middy strong wash-out is also ideal since, without spoiling successful leptogenesis, it also guarantees an independence of the initial conditions of the final asymmetry both of the initial RH neutrino abundance and of the initial value of the asymmetry. Indeed, if one assumes 
that prior to the onset of leptogenesis some external mechanism (e.g. Affleck-Dine baryogenesis, gravitational baryogenesis, GUT baryogenesis, ...) produced a large pre-existing asymmetry $N_{B-L}^{\mathrm{p}, \mathrm{i}}$, this is efficiently wash-out in way that its final relic value is exponentially suppressed as

$$
N_{B-L}^{\mathrm{p}, \mathrm{f}}=e^{-\frac{3 \pi}{8} K_{1}} N_{B-L}^{\mathrm{p}, \mathrm{i}}
$$

This optimal window for $K_{1}$ indicated by the low energy neutrino data is one of the most encouraging features of leptogenesis that somehow established its attractiveness among the many models of baryogenesis.

Within this picture one is also able to place an upper bound on the $C P$ asymmetry [7]

$$
\varepsilon_{1} \lesssim 10^{-6}\left(\frac{M_{1}}{10^{10} \mathrm{GeV}}\right) \frac{m_{\mathrm{atm}}}{m_{1}+m_{3}}
$$

that gives rise to a lower bound $M_{1} \gtrsim 10^{9} \mathrm{GeV}[7,8]$ (translating into a similar lower bound on $\left.T_{R}[7,8]\right)$ and to an upper bound $m_{1} \lesssim 0.1 \mathrm{eV}[9,6]$, showing how it is possible, thanks to the reduction of the parameters operated by the set of the assumptions of the vanilla scenario, to obtain a testable prediction. It is quite interesting that such a prediction has been now positively tested by the cosmological observations. However, within a pure generic vanilla scenario, it is impossible to go beyond such a prediction. In particular all the parameters in the leptonic mixing matrix cancel out in the total $C P$ asymmetry and, therefore, within vanilla leptogenesis there is no hope to establish any link between the matter-anti matter asymmetry and the parameters in the leptonic mixing matrix.

\section{Flavour effects}

The unflavoured assumption in the vanilla leptogenesis scenario holds only if $M_{1} \gtrsim 10^{12} \mathrm{GeV}$. Much below this particular value of the mass, the tauon lepton interactions are strong enough to measure the tauon component of the quantum lepton states produced by the decays of the RH neutrinos. For $M_{i} \ll 10^{9} \mathrm{GeV}$ also the muon interactions are strong enough and the final lepton quantum states have to be described by an incoherent mixture of electron, muon and tauon states.

In this way for $M_{1} \gtrsim 10^{12} \mathrm{GeV}$ one has a one-flavoured regime, for $10^{12} \mathrm{GeV} \gg M_{i} \gg 10^{9} \mathrm{GeV}$ one has a two-flavoured regime and for $M_{i} \ll 10^{9} \mathrm{GeV}$ one has a three-flavoured regime [10,11, 12].

It is important that one can show that there is still a lower bound $M_{1}, T_{R} \gtrsim 10^{9} \mathrm{GeV}$ for the produced asymmetry to be able to reproduced the observed one. In the two-flavour regime now the final asymmetry is given by

$$
N_{B-L}^{\mathrm{f}}=2 \varepsilon_{1} \kappa^{\mathrm{f}}\left(K_{1}\right)+\frac{1}{2} \Delta p_{1 \alpha}\left(\kappa^{\mathrm{f}}\left(K_{1 \tau}\right)-\kappa^{\mathrm{f}}\left(K_{1 \tau^{\perp}}\right)\right) .
$$

The first term is nothing else that almost the same contribution (just doubled) that we discussed in the unflavoured regime. The second term however is intrinsically very different and can dominate on the the first in many situations. One can even have $\varepsilon_{1}=0$ and still the second term can reproduce the observed asymmetry [13]. The great interest of this term is that it explicitly depends on the leptonic mixing matrix parameters. This is true not only for the additional contribution to the total $C P$ asymmetry $\Delta p_{1 \alpha}$ but also for the flavoured decay parameters $K_{i \alpha}$. In this way one can hope to 
be able to have some connection on the leptonic mixing matrix parameters. Apparently it seems that through this formula one can really give a positive answer to the two questions asked in the introduction. Unfortunately the simultaneous presence of the untested high energy parameters acts in a way that for any choice of the low energy neutrino parameters one can always chose the high energy parameters in a way that the correct asymmetry is obtained. In this way even though the low energy neutrino parameters influence the final asymmetry, without a knowledge of the high energy neutrino parameters this influence cannot be experimentally revealed, at least not within the general model independent picture we are discussing.

Therefore, not only flavour effects seem to be a missed opportunity for leptogenesis but they also seem to spoil part of the successes of the vanilla leptogenesis scenario. First of all they relax the upper bound on the neutrino masses to a level that is still undetermined. However, this relaxation does not seem to be that severe and likely a precise calculation should confirm this expectation.

At the same time flavour effects spoil the feature of vanilla leptogenesis to be easily independent of the initial conditions (it was enough to have $K_{1} \gg 1$ and this occurs naturally as we have seen). The reason is that a pre-existing asymmetry now finds easily a way to escape the RH neutrino wash-out, even when more than one RH neutrino species is included. Suppose indeed that the the asymmetry is produced with a certain flavour composition to a temperature much above the mass of the heavier thermalised RH neutrino $N_{i}$. When $T \sim M_{i}$ the wash-out from $N_{i}$ inverse processes start to become effective but the important thing is that it acts only on that component of the asymmetry along the flavour direction $\ell_{i}$, the flavour of leptons produced by the RH neutrino decays, the so called heavy neutrino flavour. RH neutrino decays cannot wash-out the orthogonal component that, therefore, escapes the wash-out. [10] If all three RH neutrino masses are above $10^{12} \mathrm{GeV}$ and they are hierarchical, then the asymmetry wash-out is sequential and the only way how a generic pre-existing asymmetry (i.e. whose flavour composition does not coincide with one of the three $\ell_{i}$ ) is washed-out completely is that the three heavy lepton flavours $\ell_{i}$ form an orthogonal basis. However, then in this case the three $\mathrm{RH}$ neutrinos do not interfere and all $C P$ asymmetries vanish. The mass pattern where all three RH neutrinos are in the three-flavoured regime $\left(M_{i} \gtrsim 10^{12} \mathrm{GeV}\right)$ cannot realise successful leptogenesis and independence of the initial conditions at the same time [14]. If RH neutrinos are assumed to be hierarchical there is only one mass pattern that can still satisfy simultaneously successful leptogenesis and at the same time guarantee independence of the initial conditions. This is the so called $N_{2}$-dominated scenario [15].

In this scenario the asymmetry is not produced by the lightest RH neutrinos but by the next-tolightest RH neutrinos. The lightest RH neutrino mass $M_{1} \ll 10^{9} \mathrm{GeV}$ is indeed too light to produce a sizeable asymmetry. However its washout occurs in the three-flavoured regime and in this way it does not suppress the asymmetry just along the $\ell_{1}$ direction but along all three charged lepton flavours directions $e, \mu, \tau$. In this was the wash-out of an asymmetry $N_{\Delta_{\alpha}}(\alpha=e, \mu, \tau)$, where $\Delta_{\alpha} \equiv B / 3-L_{\alpha}$, is described by a wash-out factor $\exp \left[-3 \pi K_{1 \alpha} / 8\right]$, where now the flavoured decay parameters $K_{1 \alpha}=p_{1 \alpha} K_{1}$, with $p_{1 \alpha}$ giving the probability that the lepton $\ell_{1}$ is measured as a charged lepton of flavour $\alpha$. Clearly in this way the wash-out along the charged lepton flavour directions is decreased and it can well happen that one $K_{1 \alpha} \lesssim 1$. This flavour has to be the tauon flavour in a way that the tauon asymmetry produced by the $N_{2} \mathrm{RH}$ neutrinos can survive and reproduce the observed asymmetry. This implies that $M_{2} \gtrsim 10^{9} \mathrm{GeV}$. On the other hand it is necessary that $K_{1 e}, K_{1 \mu} \gg 1$ in a way that the electron and muon pre-existing asymmetries (together with the possible electron 
and muon asymmetry produced by $N_{2}$ ) are washed-out. The pre-existing tauon asymmetry has to be washed-out by the $N_{2}$ wash-out processes and for this reason one also needs $M_{2} \ll 10^{12} \mathrm{GeV}$ in way that the tauon component of the pre-existing asymmetry is measured by the tauon interactions. Notice that $N_{2}$ wash out processes cannot wash-out in general the electron and muon asymmetries since these are still stored in coherent $e+\mu$ quantum superpositions. Therefore, the simultaneous request of successful leptogenesis and of independence of the initial condition, the so called strong thermal leptogenesis condition, necessarily singles out a tauon $N_{2}$-dominated scenario [14].

\section{4. $S O(10)$-inspired leptogenesis}

Flavour effects so far seem to have introduced more troubles than provided solutions. However, there is an important consequence of flavour effects that represents a way to solve a problem that is present in the vanilla scenario offering at the same time a way to realise the seemingly contrived tauon $\mathrm{N}_{2}$-dominated scenario, able to satisfy ST successful leptogenesis.

One of the main theoretical frameworks that is candidate to embed the see-saw mechanism are grand-unified $S O(10)$ models. Within these models the neutrino mass Dirac mass matrix is typically related to the mass matrices of the other fermions. For example one relation that is realised in a very simple version of $S O(10)$ models is to have $m_{D}=m_{u}$, where $m_{u}$ is the up quark mass matrix. More generally one can say that the eigenvalues of the Dirac mass matrix are not exactly equal to the up quark masses as in the simple case, but are somehow equal within $\mathscr{O}(1)$ factors. At the same time the matrix $V_{L}$ that acts on the left-handed neutrinos realising the passage from the Yukawa basis where $m_{D}$ is diagonal to the flavour basis, where the charged lepton mass matrix is diagonal, is not too different from the analogous CKM matrix in the quark sector. In particular the mixing angles are very small and the largest one is of the order of the Cabibbo angle.

Under these assumptions one can show that the RH neutrino masses are described by the following very simple expressions,

$$
M_{1} \simeq \frac{m_{D 1}^{2}}{m_{e e}} \propto m_{u}^{2}, M_{2} \simeq \frac{m_{D 2}^{2}}{m_{1} m_{2} m_{3}} \frac{m_{e e}}{\left|\left(m_{v}^{-1}\right)_{\tau \tau}\right|} \propto m_{c}^{2}, M_{3} \simeq m_{D 3}^{2}\left|\left(m_{v}^{-1}\right)_{\tau \tau}\right| \propto m_{t}^{2},
$$

where $m_{D i}$ are the eigenvalues of $m_{D}$. In particular it comes out that typically unless $m_{e e}$ and $\left(m_{v}^{-1}\right)_{\tau \tau}$ are very small, the RH neutrino masses realise exactly the $N_{2}$-dominated scenario. In addition the flavoured $C P$ asymmetries of the next-to-lightest RH neutrinos are also highly hierarchical in a way that $\varepsilon_{2 \tau} \gg \varepsilon_{2 \mu} \gg \varepsilon_{2 e}$. This results into an asymmetry that at the $N_{2}$-production is tauon dominated while the electron and the muon asymmetries are not large enough to reproduce the final asymmetry even at the production. The only way to explain the final asymmetry is then that $K_{1 \tau} \lesssim 1$, in a way that the tauon asymmetry produced by the $N_{2}$ decays survives and explains the final asymmetry realising just a tauon $N_{2}$-dominated scenario that, as we have seen, is potentially able to satisfy ST successful leptogenesis.

The formula for the final asymmetry can be expressed analytically in terms of the light neutrino mass matrix [16]. Imposing the leptogenesis bound one finds some constraints on all low energy neutrino parameters. Interestingly, for $\mathrm{NO}$, there is both a lower bound on the lightest neutrino mass $m_{1} \gtrsim 1 \mathrm{meV}$ like also an upper bound $m_{1} \lesssim 50 \mathrm{meV}$. For values $m_{1} \sim 10 \mathrm{meV}$ one also has an upper bound on the atmospheric mixing angle $\theta_{23} \lesssim 45^{\circ}$, so that the first octant is certainly 
favoured. It is also interesting that the effective $0 v \beta \beta$ neutrino mass is higher that in general and in particular for $m_{1} \gtrsim 10 \mathrm{meV}$ one has $m_{e e} \gtrsim 8 \mathrm{meV}$, testable by next generation experiments. The IO case is only marginally allowed. There is a very limited allowed regions for $10 \lesssim m_{1} \lesssim 50 \mathrm{meV}$ and for $\theta_{23} \gtrsim 46^{\circ}$. The IO case would then be completely ruled out if $\theta_{23}$ is found in the first octant.

Very interestingly $S O(10)$-inspired successful leptogenesis can also respect the ST leptogenesis condition for a subset of the solutions. In this case one obtains very definite constraints. The IO case is completely ruled out. In the NO case the lightest neutrino mass has necessarily to lie within a very narrow range $10 \lesssim m_{1} \lesssim 30 \mathrm{meV}$ testable with cosmology (it corresponds to $\left.75 \mathrm{meV} \lesssim \sum m_{i} \lesssim 125 \mathrm{meV}\right)$.

It is interesting that this ST $S O(10)$-inspired solution has correctly predicted a non-vanishing reactor mixing angle. This is visible in the left panel of Figure 2 where the ST allowed region is represented in dark blue. The solution also predicts $\delta$ in the fourth quadrant for $\theta_{23} \gtrsim 38^{\circ}$.
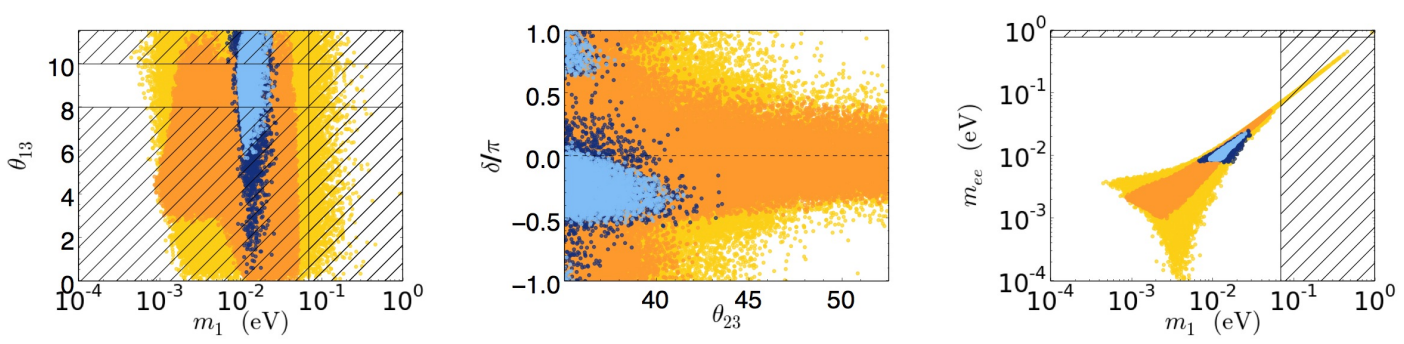

Figure 1: $S O(10)$-inspired leptogenesis solution: constraints on different planes from scatter plots. The yellow (orange) regions correspond to the case when only successful leptogenesis condition is imposed and $I \leq V_{L} \leq V_{C K M}\left(V_{L}=I\right)$. The dark (light) blue regions correspond to the case when also ST lepton genesis condition is imposed for an initial pre-existing asymmetry $N_{B-L}^{\mathrm{p}, \mathrm{i}}=10^{-3}$ (from [16]).

Interestingly current global analyses seem to indicate $\sin \delta<0$. Finally the solutions requires strictly $m_{e e} \gtrsim 8 \mathrm{meV}$ so that it predicts a signal in next generation $0 v \beta \beta$ experiments.

Table 1: Summary of the constraints from the ST SO(10)-inspired leptogenesis solution.

\begin{tabular}{|c|c|}
\hline ORDERING & NORMAL \\
\hline$m_{1}$ & $(10-30) \mathrm{meV}$ \\
\hline$\theta_{13}$ & $2^{\circ}-12^{\circ}$ \\
\hline$\delta / \pi$ & $-0.5-0$ \\
\hline$\theta_{23}$ & $\lesssim 43^{\circ}$ \\
\hline$m_{e e}$ & $(0.7-0.9) m_{1}=(7-27) \mathrm{meV}$ \\
\hline
\end{tabular}




\section{Summary}

We discussed scenario of thermal leptogenesis at a high energy scale much above the accessible scales at colliders. In this case low energy neutrino experiments are currently the only possibility to test these scenarios. In particular within $S O(10)$-inspired models one has different predictions that become quite sharp when a ST condition is also imposed the main features of this ST $S O(10)$-inspired leptogenesis solutions are summarised in the table. During next year low energy neutrino experiments will be able to fully test this solution that in any case it provides an example of how also high energy scale scenarios can be testable, though this requires condition that relate the high energy parameters to the low energy ones. It will be exciting to see whether the experiments will gradually more and more support the ST $S O(10)$-solution or whether they will rule it out. In particular the NOva experiment should soon provide some initial interesting indications in this sense.

\section{References}

[1] M. Fukugita, T. Yanagida, Phys. Lett. B 174 (1986) 45.

[2] For a recent review see S. Blanchet and P. Di Bari, New J. Phys. 14 (2012) 125012.

[3] P. Minkowski, Phys. Lett. B 67 (1977) 421; T. Yanagida, in Workshop on Unified Theories, KEK report 79-18 (1979) p. 95; M. Gell-Mann, P. Ramond, R. Slansky, in Supergravity (North Holland, Amsterdam, 1979) eds. P. van Nieuwenhuizen, D. Freedman, p. 315; S.L. Glashow, in 1979 Cargese Summer Institute on Quarks and Leptons (Plenum Press, New York, 1980) p. 687; R. Barbieri, D. V. Nanopoulos, G. Morchio and F. Strocchi, Phys. Lett. B 90 (1980) 91; R. N. Mohapatra and G. Senjanovic, Phys. Rev. Lett. 44 (1980) 912.

[4] F. Capozzi, G. L. Fogli, E. Lisi, A. Marrone, D. Montanino and A. Palazzo, arXiv:1312.2878 [hep-ph].

[5] P. A. R. Ade et al. [Planck Collaboration], arXiv:1303.5076 [astro-ph.CO].

[6] W. Buchmuller, P. Di Bari, M. Plumacher, Annals Phys. 315 (2005) 305-351.

[7] S. Davidson and A. Ibarra, Phys. Lett. B 535 (2002) 25.

[8] W. Buchmuller, P. Di Bari and M. Plumacher, Nucl. Phys. B 643 (2002) 367 [Erratum-ibid. B 793 (2008) 362]; S. Blanchet, P. Di Bari, JCAP 0703 (2007) 018.

[9] W. Buchmuller, P. Di Bari and M. Plumacher, Phys. Lett. B 547 (2002) 128 [hep-ph/0209301].

[10] R. Barbieri, P. Creminelli, A. Strumia and N. Tetradis, Nucl. Phys. B 575 (2000) 61 [hep-ph/9911315].

[11] A. Abada, S. Davidson, F. X. Josse-Michaux, M. Losada and A. Riotto, JCAP 0604 (2006) 004 [hep-ph/0601083].

[12] S. Blanchet, P. Di Bari and G. G. Raffelt, JCAP 0703 (2007) 012 [hep-ph/0611337].

[13] E. Nardi, Y. Nir, E. Roulet and J. Racker, JHEP 0601 (2006) 164 [hep-ph/0601084].

[14] E. Bertuzzo, P. Di Bari, L. Marzola, Nucl. Phys. B849 (2011) 521-548.

[15] P. Di Bari, Nucl. Phys. B727 (2005) 318-354; O. Vives, Phys. Rev. D73 (2006) 073006; S. Blanchet and P. Di Bari, Nucl. Phys. B 807 (2009) 155.

[16] P. Di Bari, L. Marzola and M. R. Fiorentin, arXiv:1411.5478 [hep-ph]. 